\title{
Does Fast Adaptive Modulation Always Outperform Slow Adaptive Modulation?
}

\author{
Laura Toni, Member, IEEE, and Andrea Conti, Senior Member, IEEE
}

\begin{abstract}
Link adaptation techniques are important modern and future wireless communication systems to cope with quality of service fluctuations in fading channels. These techniques require the knowledge of the channel state obtained with a portion of resources devoted to channel estimation instead of data and updated every coherence time of the process to be tracked. In this paper, we analyze fast and slow adaptive modulation systems with diversity and non-ideal channel estimation under energy constraints. The framework enables to address the following questions: (i) What is the impact of non-ideal channel estimation on fast and slow adaptive modulation systems? (ii) How to define a proper figure of merit which considers both resources dedicated to data and those to channel estimation? (iii) Does fast adaptive always outperform slow adaptive techniques? Our analysis shows that, despite the lower complexity and feedback rate, slow adaptive modulation (SAM) can achieve higher spectral efficiency than fast adaptive modulation (FAM) in the presence of energy constraint, diversity, and non-ideal channel estimation. In addition, SAM satisfies bit error outage requirements also in FAM-denied region.
\end{abstract}

Index Terms-Adaptive modulation, multichannel reception, channel estimation, fading channels, performance evaluation.

\section{INTRODUCTION}

$\mathbf{T}$ HE diffusion of high speed digital wireless communications has increased the need for reliable high data rate communications in variable channel conditions. Adaptive modulation techniques allow to maximize the spectral efficiency (SE) in fading channels without compromising the performance in terms of bit error probability (BEP) and bit error outage (BEO) (see, e.g., [1]-[7]). The $M$-ary quadrature amplitude modulation ( $M$-QAM) achieves high SE and it is widely considered in adaptive modulation systems. In [3], for example, power and rate were both adapted to channel conditions for a $M$-QAM uncoded system. The gain derived from an adaptive rather than a fixed transmission scheme is reported, and it was shown that the channel capacity adapting only the data rate is almost the same of adapting both rate and power. The fast adaptive modulation (FAM) technique tracks instantaneous channel variations due to small-scale fading; the receiver estimates the instantaneous signal-to-noise ratio (SNR) and send a feedback to the transmitter with the optimal

Manuscript received April 19, 2010; revised November 26, 2010; accepted January 8, 2011. The associate editor coordinating the review of this paper and approving it for publication was A. Yener.

This research was supported in part by the FP7 European Network of Excellence NEWCom++ grant.

L. Toni is with TERA, Italian Institute of Technology (IIT), Via Morego, 3016163 Genova, Italy, and with WiLab (e-mail: laura.toni@iit.it).

A. Conti is with ENDIF, University of Ferrara, Via Saragat, 144100 Ferrara, Italy, and with WiLab at University of Bologna, Viale Risorgimento 2, 40136 Bologna, Italy (e-mail: a.conti@ieee.org).

Digital Object Identifier 10.1109/TWC.2011.020111.100643 constellation signaling to be used, [1]-[4]. Through adaptation, good channel conditions are exploited increasing the transmitted throughput and, at the same time, preserving the performance in case of bad channel conditions. In [5], a slow adaptive modulation (SAM) technique has been proposed, in which the constellation signaling is adapted tracking largescale channel variations (averaged over the small-scale fading) and compared to FAM. The analysis applies for systems employing diversity and operating in small-scale and largescale fading channels with ideal channel estimation.

Typical performance metrics for adaptive communication systems are the BEP, the BEO (i.e., the BEP-based outage probability [8]), and the SE. It is worth noting that FAM leads to best performance at the cost of a frequent channel estimation or prediction and high feedback rate. For a given target BEP, the SE and BEO achieved by SAM are close to that of FAM and show a significant improvement with respect to a fixed modulation scheme. With respect to FAM, the SAM technique requires a reduced feedback rate and has lower complexity.

For both FAM and SAM techniques, an important role is played by the channel estimation. The effects of outdated channel estimation are investigated for adaptive modulation systems in [3]. Adaptive modulation systems with non-ideal channel estimation for single- and multi-carrier systems with FAM are analyzed in [3], [6], [9]-[11]. Channel estimation techniques typically utilize resources that would be devoted to data transmission (e.g., pilot symbols can be inserted during the transmission of data symbols) thus sacrificing the SE. Hence, it is important to define proper figure of merit able to capture the trade-off between quality of service and resource utilization depending on the amount of energy devoted to data and pilots.

In this paper, we analyze slow and fast adaptive $M$-QAM systems with diversity accounting for resources dedicated to non-ideal channel estimation. The contribution is three-fold: (i) to define the achieved SE (ASE) which enables to take into account the portion of transmitted frame dedicated to data and pilot symbols; (ii) to analyze SAM with diversity in the presence of non-ideal CSI under energy constraints; (iii) to compare FAM and SAM under various conditions and constraints. The framework developed enables the design of adaptive diversity systems under energy constraints and nonideal channel estimation.

The reminder of this paper is organized as follows. In Section II the system model and assumptions are presented and in Section III the performance is derived and a new metric which considers also the resources utilized for channel estimation is 


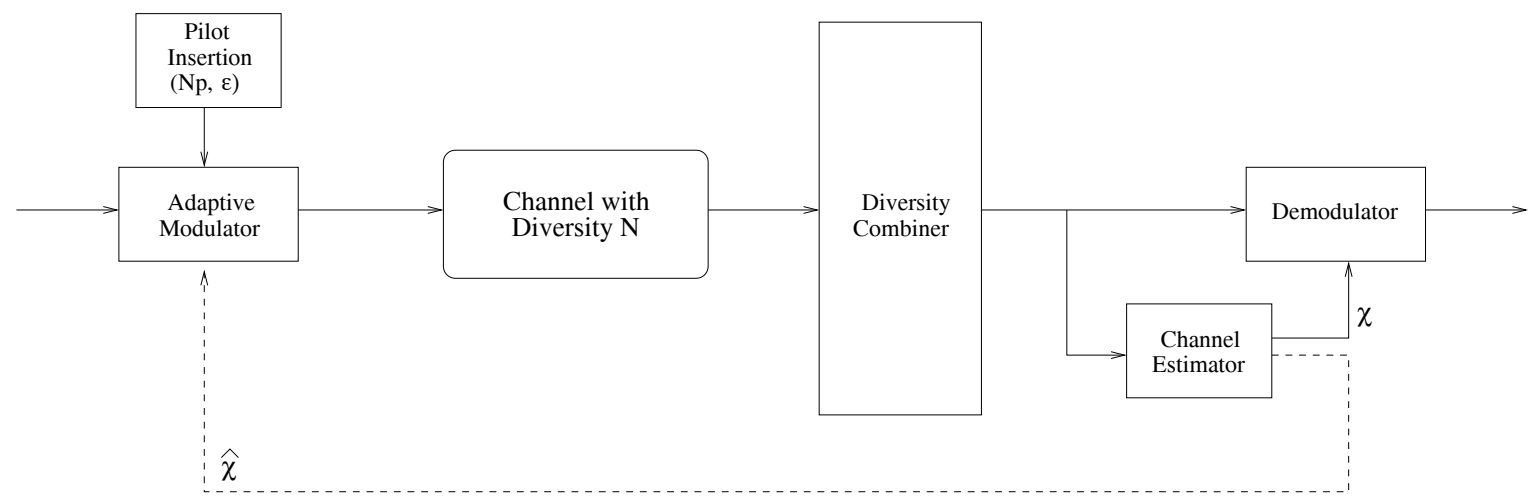

Fig. 1. Block scheme of the considered adaptive communication system ( $\chi$ assumes different meanings depending on the adaptive modulation technique).

defined. In Section IV the performance is evaluated in terms of BEP, BEO, and the new definition of SE under constraints. In Section $\mathrm{V}$ numerical results are given with indications on how they can be utilized by a system designer. Finally, our conclusions are given in Section VI.

\section{System MOdel And ASSUMPTIONS}

We consider an adaptive modulation system (see Fig. 1) with $M$-QAM squared constellation signaling in composite Rayleigh fading and log-normal shadowing over $N_{\mathrm{d}}$-branch multichannel reception with maximal ratio diversity (MRD). Independent, identically distributed (i.i.d.) fading and same shadowing level over all branches, (i.e., microdiversity) is considered. ${ }^{1}$ We denote by $h_{k}$ the small-scale fading gain on the $k$-th branch which is distributed as a complex Gaussian random variable (RV) with mean zero and variance $\sigma_{\mathrm{h}}^{2}$ per dimension for all branches.

We consider discrete variable-rate modulation schemes where a set of $J+1$ constellation signaling $\left\{M_{0}, M_{1}, \ldots, M_{J}\right\}$ can be adopted. As an example, digital video broadcasting system employs $M \in\{4,16,64,256\}$, then $M_{j}=4^{j+1}$ and $J=3$. The constellation signaling is chosen opportunistically depending on the value of a quantity $\chi$ which is, respectively, the instantaneous SNR $\gamma$ in the case of FAM and the mean $\operatorname{SNR} \bar{\gamma}$, averaged over small-scale fading, for SAM. Given a target BEP $P_{\mathrm{b}}^{\star}$, the required SNR for the modulation $M_{j}$ is $\chi_{j}^{\star}$ such that $P_{\mathrm{b}}\left(\chi_{j}^{\star}\right)=P_{\mathrm{b}}^{\star}$. The opportunistic modulation is chosen by comparing the estimated SNR value with the SNRs required for each modulation to satisfy the target BEP. When the SNR value falls in the region $\left[\chi_{j}^{\star}, \chi_{j+1}^{\star}\right)$, the $j$-th constellation size $M_{j}$ is adopted.

We consider a pilot symbols assisted modulation (PSAM) scheme (see, e.g., [12]-[16]) where the transmitted frame is composed by $N_{\mathrm{s}}$ data symbols (each with mean energy $E_{\mathrm{s}}$ ) and $N_{\mathrm{p}}$ pilot symbols for channel estimation (each with mean energy $\left.E_{\mathrm{p}}=\varepsilon E_{\mathrm{s}}\right){ }^{2}$ The mean SNR on each diversity branch is $\bar{\gamma}=\mathbb{E}\left\{|h|^{2}\right\} E_{\mathrm{s}} / N_{0}$, where $N_{0} / 2$ is the two-sided spectral density of the additive white Gaussian noise (AWGN)

\footnotetext{
${ }^{1}$ Since we consider microdiversity, in the following we will omit the branch subscript in the notation of the mean SNR, averaged over small-scale fading.

${ }^{2}$ The frame is structured to transmit $N_{\mathrm{p}}$ pilots within a coherence time of the channel.
}

and $\mathbb{E}\{\cdot\}$ denotes the statistical expectation (here evaluated over the small-scale fading). The shadowing is assumed lognormal distributed where $\bar{\gamma}_{\mathrm{dB}}=10 \log _{10} \bar{\gamma}$ is a Gaussian RV having mean $\mu_{\mathrm{dB}}$ and variance $\sigma_{\mathrm{dB}}^{2}$. The channel estimator is maximum-likelihood and fading channels over branches are such that $\mathbf{h}=\left[h_{1} h_{2} \ldots h_{N_{\mathrm{d}}}\right]$ is constant over a frame, and independent frame by frame (block fading channel [17]-[19]). The estimated fading gain on the $k$-th branch is

$$
\widehat{h}_{k}=h_{k}+e_{k}
$$

where $e_{k}$ is a zero mean complex Gaussian RV with variance per dimension $\sigma_{\mathrm{e}}^{2}$. In [13] the $\sigma_{\mathrm{e}}^{2}$ is derived as a function of the pilot symbols energy and noise spectral density, as given by

$$
\sigma_{\mathrm{e}}^{2}=\frac{N_{0}}{2 N_{\mathrm{p}} E_{\mathrm{p}}} .
$$

For $M$-QAM adaptive modulation systems, the non-ideal channel estimation affects both the transmitter side (in the choice of the opportunistic constellation signaling) and the receiver side (in diversity combining and bit reconstructing). To adapt the constellation size to the most updated channel estimation, tight delay constraints should be met in the evaluation of the channel state information (CSI) used at the transmitter side. Hence, for each frame the $N_{\mathrm{p}}$ received pilot symbols are processed for the channel estimation, which is sent over an error-free feedback channel. Since the CSI employed at the receiver side does not have such a tight delay constraint, a longer interpolation involving a larger set of pilot symbols can be harnessed at the receiver, leading to a more reliable CSI than the one at the transmitter side. It follows that the channel estimate at the transmitter can be less accurate than that at the receiver (see, e.g., [3]). In the following we assume non-ideal CSI at the transmitter side and an ideal CSI at the receiver side. Note that, for the effects of delayed channel estimates (outdated channels) the reader may refer to, e.g., [3], [11], here we focus on updated but erroneous CSI feedback.

In addition to the analytical results, evaluated based on the block fading model discussed above, we also show simulative results based on both the block fading (to verify the analysis) and the time-varying models. In particular, to simulate the time-varying small-scale fading, we assume Rayleigh fading and the modified Jakes' model [20], [21]. 


\section{Performance Metrics with Non-Ideal CHANNEL ESTIMATION}

For a given target BEP, typical performance metrics for adaptive modulation systems are the SE and the BEO. We evaluate them for multichannel communications with nonideal channel estimation. For each possible constellation signaling, the SNR value required to reach the target BEP is evaluated and compared to the estimated SNR value $\hat{\chi}$ (i.e., $\widehat{\chi}_{\text {FAM }}$ for FAM and $\hat{\chi}_{\text {SAM }}$ for SAM). The mean SE and the BEO for systems affected by channel estimation errors can be evaluated from the probability density function (PDF) or the cumulative distribution function (CDF) of $\widehat{\chi}$, that we now evaluate for FAM and SAM systems.

The performance of FAM systems depends on the estimated instantaneous SNR at the combiner output $\widehat{\gamma}_{\mathrm{T}}$. For MRD $\widehat{\chi}_{\mathrm{FAM}}$ is then given by

$$
\widehat{\chi}_{\text {FAM }}=\widehat{\gamma}_{\mathrm{T}}=\sum_{k=1}^{N_{\mathrm{d}}} \widehat{\gamma}_{k}=\sum_{k=1}^{N_{\mathrm{d}}}\left|\widehat{h}_{k}\right|^{2} \frac{E_{\mathrm{s}}}{N_{0}} .
$$

Both real and imaginary parts of $\widehat{h}_{k}$ are zero-mean Gaussian distributed with variance $\sigma_{\mathrm{h}}^{2}+\sigma_{\mathrm{e}}^{2}$. Therefore, the PDF of the estimated instantaneous SNR $\widehat{\gamma}_{\mathrm{T}}$, conditioned to the mean SNR per branch $\bar{\gamma}$, is a chi-square distribution

$$
f_{\widehat{\gamma}_{\mathrm{T}} \mid \bar{\gamma}}(\xi)=\frac{\xi^{N_{\mathrm{d}}-1}}{\bar{\gamma}^{N_{\mathrm{d}}} \sigma_{t}^{2 N_{\mathrm{d}}} \Gamma\left(N_{\mathrm{d}}\right)} \exp \left[-\frac{\xi}{\bar{\gamma} \sigma_{\mathrm{t}}^{2}}\right]
$$

for $\xi \geq 0$ and 0 otherwise, where $\sigma_{\mathrm{t}}^{2}=1 / \rho^{2}$ and

$$
\begin{aligned}
\rho & =\frac{\left(\mathbb{E}\left\{h_{k} \widehat{h}_{k}^{*}\right\}-\mathbb{E}\left\{h_{k}\right\} \mathbb{E}\left\{\widehat{h}_{k}^{*}\right\}\right)}{\sqrt{\mathbb{E}\left\{\left|h_{k}-\mathbb{E}\left\{h_{k}\right\}\right|^{2}\right\} \mathbb{E}\left\{\left|\widehat{h}_{k}-\mathbb{E}\left\{\widehat{h}_{k}\right\}\right|^{2}\right\}}} \\
& =\frac{\mathbb{E}\left\{h_{k} \widehat{h}_{k}^{*}\right\}}{\sqrt{\mathbb{E}\left\{\left|h_{k}\right|^{2}\right\} \mathbb{E}\left\{\left|\widehat{h}_{k}\right|^{2}\right\}}} \\
& =\sqrt{\frac{\sigma_{\mathrm{h}}^{2}}{\sigma_{\mathrm{h}}^{2}+\sigma_{\mathrm{e}}^{2}}}=\frac{N_{\mathrm{p}} \varepsilon}{N_{\mathrm{p}} \varepsilon+\frac{1}{\bar{\gamma}}}
\end{aligned}
$$

is the envelope of the complex correlation between $h_{k}$ and $\widehat{h}_{k}$ [13]. In (5), $\varepsilon=E_{\mathrm{p}} / E_{\mathrm{s}}$ and the second equality follows from $\mathbb{E}\left\{h_{k}\right\}=\mathbb{E}\left\{\widehat{h}_{k}^{*}\right\}=\mathbb{E}\left\{e_{k}\right\}=0$. From (4), the marginal PDF of the estimated instantaneous SNR is derived as $f_{\widehat{\gamma}_{\mathrm{T}}}(\xi)=\int f_{\widehat{\gamma}_{T} \mid \bar{\gamma}}(\xi) f_{\bar{\gamma}}(w) d w$, and the CDF can be easily obtained. For log-normal shadowing the PDF of the mean branch SNR is given by

$$
f_{\bar{\gamma}}(w)=\frac{\nu}{\sqrt{2 \pi} \sigma_{\mathrm{dB}} w} \exp \left[-\frac{\left(10 \log _{10} w-\mu_{\mathrm{dB}}\right)^{2}}{2 \sigma_{\mathrm{dB}}^{2}}\right]
$$

for $w \geq 0$ and 0 otherwise, with $\nu=10 / \ln 10$.

The performance of SAM systems depends on the estimated mean SNR $\widehat{\chi}_{\text {SAM }}=\breve{\gamma}$ which is given by ${ }^{3}$

$$
\widehat{\chi}_{\mathrm{SAM}}=\breve{\gamma}=\mathbb{E}\left\{\widehat{\gamma}_{k}\right\}=2\left(\sigma_{\mathrm{e}}^{2}+\sigma_{\mathrm{h}}^{2}\right) \frac{E_{\mathrm{s}}}{N_{0}}=\sigma_{\mathrm{t}}^{2} \bar{\gamma}=\bar{\gamma}+\frac{1}{N_{\mathrm{p}} \varepsilon} .
$$

From (6) and (7), the CDF of the estimated mean SNR results in

$$
F_{\breve{\gamma}}(x)=Q\left(\frac{\mu_{\mathrm{dB}}-10 \log _{10}\left(x-\frac{1}{N_{\mathrm{p}} \varepsilon}\right)}{\sigma_{\mathrm{dB}}}\right)
$$

for $x \geq 1 /\left(N_{\mathrm{p}} \varepsilon\right)$ and 0 otherwise, where $Q(x)=\int_{x}^{\infty} e^{-t^{2} / 2} d t$ is the Gaussian- $Q$ function. For simplicity of notation, in the following we will write $\widehat{\chi}$ to represent $\hat{\chi}_{\text {FAM }}$ or $\widehat{\chi}_{\text {SAM }}$ for FAM or SAM systems, respectively.

\section{A. Bit Error Outage}

The BEO is an important performance metric for digital communication systems which is defined as the probability that the BEP is greater than the target BEP [8], [22]-[24], that is

$$
P_{\mathrm{o}}\left(P_{\mathrm{b}}^{\star}\right)=\mathbb{P}\left\{P_{\mathrm{b}}(\chi)>P_{\mathrm{b}}^{\star}\right\} .
$$

The system is in outage when even the constellation size $M_{0}$, which is the more robust against disturbances, does not fulfill the BEP requirement or when the increasing of $M$ is dictated by an erroneous channel estimation. For FAM the exact instantaneous BEP expression is given by (10), reported at the bottom of this page [25], [26] whereas for SAM the exact mean BEP expression is given by (11), reported at the bottom of this page, where $\lfloor x\rfloor$ denotes the largest integer less than or equal to $x$, and

$$
I_{N_{\mathrm{d}}}(\bar{\gamma})=\frac{1}{\pi} \int_{0}^{\pi / 2}\left[\frac{\sin ^{2}(\theta)}{\sin ^{2}(\theta)+\frac{3(2 i+1)^{2}}{2(M-1)} \bar{\gamma}}\right]^{N_{\mathrm{d}}} \mathrm{d} \theta .
$$

${ }^{3}$ Note that $\breve{\gamma}=\bar{\gamma}$ for ideal channel estimation.

$$
\begin{aligned}
& P_{\mathrm{b}}\left(\gamma_{\mathrm{T}}\right)=\frac{2}{\sqrt{M} \log _{2}(\sqrt{M})} \sum_{h=1}^{\log _{2}(\sqrt{M})} \sum_{i=0}^{\left(1-2^{-h}\right) \sqrt{M}-1}(-1)^{\left\lfloor\frac{i 2^{h-1}}{\sqrt{M}}\right\rfloor}\left(2^{h-1}-\left\lfloor\frac{i 2^{h-1}}{\sqrt{M}}+\frac{1}{2}\right\rfloor\right) Q\left((2 i+1) \sqrt{\frac{3 \gamma_{\mathrm{T}}}{(M-1)}}\right) \\
& P_{b}(\bar{\gamma})=\int_{0}^{\infty} P_{\mathrm{b}}\left(\gamma_{\mathrm{T}}\right) f_{\gamma_{\mathrm{T}} \mid \bar{\gamma}}(\xi) d \xi=\frac{2}{\sqrt{M} \log _{2}(\sqrt{M})} \sum_{h=1}^{\log _{2}(\sqrt{M})} \sum_{i=0}^{\left(1-2^{-h}\right) \sqrt{M}-1}(-1)^{\left\lfloor\frac{i 2^{h-1}}{\sqrt{M}}\right\rfloor} \\
& \times\left(2^{h-1}-\left\lfloor\frac{i 2^{h-1}}{\sqrt{M}}+\frac{1}{2}\right\rfloor\right) \underbrace{\int_{0}^{\infty} Q\left((2 i+1) \sqrt{\frac{3 \gamma_{\mathrm{T}}}{(M-1)}}\right) f_{\gamma_{\mathrm{T}} \mid \bar{\gamma}}(\xi) d \xi}_{I_{N_{\mathrm{d}}}(\bar{\gamma})}
\end{aligned}
$$


In the case of ideal channel estimation, the BEO is given by

$$
P_{\mathrm{o}}\left(P_{\mathrm{b}}^{\star}\right)=F_{\chi}\left(\chi_{0}^{\star}\right)
$$

where $F_{\chi}(\cdot)$ is the CDF of $\chi$, being $\chi=\gamma_{\mathrm{T}}$ for FAM and $\chi=\bar{\gamma}$ for SAM [5],

In systems with non-ideal CSI at the transmitter, the estimated SNR $\hat{\chi}$ can be an underestimate or an overestimate of the true value. The former case leads to a reduction of the $\mathrm{SE}$ and the BEO, while the latter leads to an increase of the SE and BEO. In particular, when $\hat{\chi}>\chi$, although the true SNR would fall within the range for the $j$-th modulation, the modulation $M_{j+1}$ might be adopted. In this case, the BEP can be greater than the target BEP and the system is in outage.

Thus, the system is in outage when $\chi<\chi_{0}^{\star}$ or $\widehat{\chi}>\chi_{i}^{\star}$ and $\chi \leq \chi_{i}^{\star}$ for all $i$. Therefore, for $\hat{\chi}=\chi+\Delta \chi$, the outage occurs for $\chi_{i}^{\star}-\Delta \chi<\chi \leq \chi_{i}^{\star}$ or $\chi<\chi_{0}^{\star}$ and the conditioned BEO $P_{\mathrm{o} \mid \Delta \chi}$ results to be

$\left\{\begin{array}{l}F_{\chi}\left(\chi_{0}^{\star}\right)+\sum_{j=1}^{J}\left[F_{\chi}\left(\chi_{j}^{\star}\right)-F_{\chi}\left(\chi_{j}^{\star}-\Delta \chi\right)\right] \\ F_{\chi}\left(\chi_{0}^{\star}\right)\end{array}\right.$

with $\Delta \chi \geq 0$ otherwise.

For FAM systems, $\Delta \chi=\Delta \gamma_{\mathrm{T}}$ is a RV whose $\mathrm{PDF} f_{\Delta \chi}(\cdot)$ is derived in Appendix and the unconditioned BEO is given by

$$
P_{\mathrm{o}}=\int P_{\mathrm{o} \mid \Delta \chi} f_{\Delta \chi}(\xi) d \xi
$$

For SAM systems, we note from (7) that $\Delta \chi=1 /\left(N_{\mathrm{p}} \varepsilon\right)$ is deterministic and

$$
P_{\mathrm{o}}=Q\left(a_{0}\right)+\sum_{j=1}^{J}\left[Q\left(a_{j}\right)-Q\left(b_{j}\right)\right],
$$

where

$$
\begin{aligned}
a_{j} & =\frac{\mu_{\mathrm{dB}}-\bar{\gamma}_{j, \mathrm{~dB}}^{*}}{\sigma_{\mathrm{dB}}} \\
b_{j} & =\frac{\mu_{\mathrm{dB}}-10 \log _{10}\left(\bar{\gamma}_{j}^{*}-\frac{1}{N_{\mathrm{p}} \varepsilon}\right)}{\sigma_{\mathrm{dB}}} .
\end{aligned}
$$

In the absence of energy constraint, it is expected that, by increasing $N_{\mathrm{p}} \varepsilon$, the channel estimation accuracy increases and the BEO approaches the one for ideal CSI. We compare the case of non-ideal channel estimation with the ideal one, by evaluating the penalty on the required median SNR for a given target BEO and BEP. We recall that adaptive systems with ideal CSI at the receiver side are in outage when even the most robust constellation size (i.e., $M=M_{0}$ ) does not meet the target BEP. In the presence of small- and large-scale fading, the $\mathrm{BEO}$ is a function of the median SNR value $\mu_{\mathrm{dB}}$. Given a target $\mathrm{BEO} P_{\mathrm{o}}^{\star}, \mu_{\mathrm{dB}, 0}$ denotes the median SNR value which reach $P_{\mathrm{o}}=P_{\mathrm{o}}^{\star}$ when the lowest constellation size is adopted, that is $M=M_{0}$. In the case of non-ideal CSI, the system is in outage not only when the lowest constellation size does not meet the target BEP, but also when the SNR in the feedback is overestimated. In this case, the system might switch to a modulation level higher than the best one, and the system might experience a BEP greater than the target one. Here we take into account both the effects causing the BEO, and we denote $\widehat{\mu}_{\mathrm{dB}, 0}$ as the median SNR value which reaches the target BEO for non-ideal channel estimation. Then, the median SNR penalty for a given BEO is defined as

$$
\Delta \mu_{\mathrm{dB}} \triangleq \widehat{\mu}_{\mathrm{dB}, 0}-\mu_{\mathrm{dB}, 0}
$$

which represents the increasing in required median SNR for non-ideal CSI with respect to the case of ideal CSI.

\section{B. Achieved Spectral Efficiency}

The available SE is given by $\log _{2} M_{J}$ which would be reached if channel conditions are such that the system is always in service with the greatest constellation size. In the presence of non-ideal channel estimation and outage events, the ASE (i.e., the SE achieved considering resources dedicated to channel estimation) might be lower than the available SE and its characterization is important for system design. The mean $\mathrm{SE}[\mathrm{bps} / \mathrm{Hz}]$ with ideal channel estimation is given by[5]

$$
\eta=\sum_{j=0}^{J-1} \widetilde{M}_{j}\left[F_{\chi}\left(\chi_{j+1}^{\star}\right)-F_{\chi}\left(\chi_{j}\right)\right]+\widetilde{M}_{J}\left[1-F_{\chi}\left(\chi_{J}^{\star}\right)\right],
$$

where $\widetilde{M}_{j}=\log _{2} M_{j}$.

In the case of non-ideal channel estimation, the SNR $\chi$ is replaced by the estimated one $\hat{\chi}$. The insertion of pilot symbols occupies part of the resources that could otherwise be utilized for data symbols. In each frame of length $N_{\text {tot }}$ symbols, $N_{\mathrm{p}}$ pilot symbols and $N_{\mathrm{s}}=N_{\text {tot }}-N_{\mathrm{p}}$ data symbols are transmitted. By denoting the fraction dedicated to pilots and data as $n_{\mathrm{p}}=N_{\mathrm{p}} / N_{\text {tot }}$ and $n_{\mathrm{s}}=N_{\mathrm{s}} / N_{\text {tot }}$, respectively, we define the ASE as

$$
\eta_{\mathrm{A}} \triangleq \eta n_{\mathrm{s}}=\eta \frac{\left(N_{\mathrm{tot}}-N_{\mathrm{p}}\right)}{N_{\text {tot }}} .
$$

For MRD the ASE implicitly depends on the number of branches, $N_{\mathrm{d}}$. By increasing $N_{\mathrm{d}}$, the BEP is reduced, and the SNR thresholds decreases, leading in general to a greater SE. In the case of subset diversity (SSD), where $L$ branches out of $N_{\mathrm{d}}$ are selected, $\left\lceil N_{\mathrm{d}} / L\right\rceil N_{\mathrm{p}}$ pilots should be transmitted to guarantee $N_{\mathrm{p}}$ received symbols per branch [27]-[30], where $\lceil x\rceil$ denotes the largest integer higher than or equal to $x$. Then, the ASE would result in $\eta_{\mathrm{A}} \triangleq \eta\left(N_{\text {tot }}-\left\lceil N_{\mathrm{d}} / L\right\rceil N_{\mathrm{p}}\right) /\left(N_{\text {tot }}\right)$, which explicitly depends on $L$ and $N_{\mathrm{d}}$.

We also define the mean spectral efficiency penalty as the ratio of the SE evaluated with ideal CSI $\eta^{\text {(ideal) }}$ and the ASE evaluated in the non-ideal CSI case $\eta_{\mathrm{A}}$ as

$$
\Delta \eta \triangleq \frac{\eta^{(\text {ideal })}}{\eta_{\mathrm{A}}} .
$$

Obviously, with ideal channel estimation $\Delta \eta=1$. We recall that for SAM systems, the coherence time of tracked channel variation is greater than the one for FAM, and for a given amount of resources dedicated to the channel estimation, the portion of data symbols per frame is greater than the one for FAM, thus $n_{\mathrm{s}}^{\text {FAM }} \leq n_{\mathrm{s}}^{\mathrm{SAM}}$. Conversely, for a given value of $n_{\mathrm{p}}$, the number of pilot symbols dedicated to channel estimation is greater for SAM than for FAM.

\section{Performance Analysis Under Constraints}

In adaptive modulation systems with non-ideal CSI, the performance strictly depends on the pilot scheme adopted. In the following, we analyze the effects of both the imposed 
constraints and the pilot scheme design on the system performance. In both FAM and SAM systems, the following constraints are imposed over each frame

$$
\left\{\begin{array}{l}
N_{\mathrm{p}}+N_{\mathrm{s}}=N_{\text {tot }} \\
N_{\mathrm{p}} E_{\mathrm{p}}+N_{\mathrm{s}} E_{\mathrm{s}}=E_{\text {tot }} .
\end{array}\right.
$$

Then, the energy dedicated to data depends on the adopted pilot scheme as

$$
E_{\mathrm{s}}=\frac{E}{\frac{N_{\mathrm{p}}}{N_{\mathrm{tot}}}(\varepsilon-1)+1}
$$

where $E \triangleq E_{\text {tot }} / N_{\text {tot }}$. Note that the increasing of $N_{\mathrm{p}} \varepsilon$ leads to a better channel estimation and to a lowering of $E_{\mathrm{s}}$, the two situations have opposite effects on the system performance. Consequently, even the exact SNR $\chi$ is a function of the pilot scheme. This energy partition problem was investigated in [16] for cellular systems.

\section{A. FAM Systems}

From the above constraints, the SNR $\chi=\gamma_{\mathrm{T}}$ can be expressed as

$$
\begin{aligned}
\gamma_{\mathrm{T}} & =\sum_{k=1}^{N_{\mathrm{d}}} \frac{\left|h_{k}\right|^{2}}{N_{0}} \frac{E}{\frac{N_{\mathrm{p}}}{N_{\mathrm{tot}}}(\varepsilon-1)+1} \\
& =\frac{v_{\mathrm{T}}}{\frac{N_{\mathrm{p}}}{N_{\mathrm{tot}}}(\varepsilon-1)+1}
\end{aligned}
$$

where $v_{\mathrm{T}} \triangleq \sum_{k=1}^{N_{\mathrm{d}}}\left|h_{k}\right|^{2} E / N_{0}$. By substituting (23) in the BEP expression $P_{\mathrm{b}}(\chi)$ reported in (10), the $P_{\mathrm{b}}$ results a function of two parameters: $N_{\mathrm{p}} \varepsilon$ which characterizes the pilot scheme design and $v_{\mathrm{T}}$ which represents the SNR per generic (pilot or data) symbol. Thus, through (10) the instantaneous $\mathrm{BEP}$ is

$$
\begin{aligned}
P_{\mathrm{b}}\left(v_{\mathrm{T}}, N_{\mathrm{p}} \varepsilon, M_{j}\right)=P_{\mathrm{b}}\left(\gamma_{\mathrm{T}}\right), \text { with } \gamma_{\mathrm{T}} & =\frac{v_{\mathrm{T}}}{\frac{N_{\mathrm{p}}}{N_{\mathrm{tot}}}(\varepsilon-1)+1} \\
\text { for } M & =M_{j}
\end{aligned}
$$

Unlike the SNR $\gamma_{\mathrm{T}}$, the $v_{\mathrm{T}}$ does not depend on the pilot scheme, but only on the mean energy over the frame, the $N_{0}$, and the channel conditions. Therefore, we will compare systems with different pilot schemes for a given $v_{\mathrm{T}}$. When constraints are imposed, $v_{\mathrm{T}}$ is the SNR variable in the BEP expression based on which the constellation size is chosen (for $\varepsilon=1$, then $\left.v_{\mathrm{T}} \rightarrow \gamma_{\mathrm{T}}\right)$. It means that the $j$-th SNR threshold $\left(M=M_{j}\right)$ is defined as

$$
v_{\mathrm{T}, j}^{\star} \text { such that } P_{\mathrm{b}}\left(v_{\mathrm{T}, j}^{\star}, N_{\mathrm{p}} \varepsilon, M_{j}\right)=P_{\mathrm{b}}^{\star} .
$$

Due to the imposed constraints, a double effect of the pilot assisted channel estimation is present: i) parameters $N_{\mathrm{p}}$ and $\varepsilon$ affect the data energy, leading to an increase of the thresholds levels $v_{\mathrm{T}, j}^{\star}$; ii) the accuracy of the estimation in the feedback channel depends on $N_{\mathrm{p}}$ and $\varepsilon$. In particular, the feedback estimated SNR is

$$
\widehat{v}_{\mathrm{T}}=\sum_{k=1}^{N_{\mathrm{d}}}\left|\widehat{h}_{k}\right|^{2} \frac{E}{N_{0}}
$$

where only $\widehat{h}_{k}$ depends on the pilot scheme. When ideal systems (ideal CSI without constraints) are considered, $\widehat{v}_{\mathrm{T}}$ corresponds to $\gamma_{\mathrm{T}}$.

In FAM systems with energy and symbols constraints, the BEO can be evaluated from (9) and (14) for ideal and nonideal CSI respectively, with $\chi=v_{\mathrm{T}}, \chi_{j}^{\star}=v_{\mathrm{T}, j}^{\star}, \Delta \chi=\Delta v_{\mathrm{T}}$, and $F_{\chi}(\xi)=F_{v_{\mathrm{T}}}(\zeta)$, being $F_{v_{\mathrm{T}}}(\zeta)$ the CDF of $v_{\mathrm{T}}$. The ASE can still be evaluated from (18) and (19), with $F_{\chi}(\xi)=$ $F_{\widehat{v}_{\mathrm{T}}}(\zeta)$. The CDF of $\widehat{v}_{\mathrm{T}}$ can be derived from the marginal PDF of $f_{\widehat{v}_{\mathrm{T}}}(\zeta)=\int f_{\widehat{v}_{\mathrm{T}} \mid \bar{v}}(\zeta) f_{\bar{v}}(w) d w$, where the conditional $\mathrm{PDF}$ is

$$
f_{\widehat{v}_{\mathrm{T}} \mid \bar{v}}(\zeta)=\frac{\zeta^{N_{\mathrm{d}}-1}}{\bar{v}^{N_{\mathrm{d}}} \sigma_{\mathrm{t}}^{2 N_{\mathrm{d}}} \Gamma\left(N_{\mathrm{d}}\right)} \exp \left[-\frac{\zeta}{\bar{v} \sigma_{\mathrm{t}}^{2}}\right],
$$

for $\zeta \geq 0$, and 0 otherwise.

\section{B. SAM Systems}

In SAM systems, the same considerations of the FAM case hold, but based on quantities averaged over the small-scale fading. The mean SNR is given by

$$
\begin{aligned}
\bar{\gamma} & =\frac{\mathbb{E}\left\{|h|^{2}\right\}}{N_{0}} \frac{E}{\frac{N_{\mathrm{p}}}{N_{\mathrm{tot}}}(\varepsilon-1)+1} \\
& =\frac{\bar{v}}{\frac{N_{\mathrm{p}}}{N_{\mathrm{tot}}}(\varepsilon-1)+1}
\end{aligned}
$$

where $\bar{v} \triangleq \mathbb{E}\left\{|h|^{2}\right\} E / N_{0}$. In the degenerative case of $\varepsilon=1$, we have that $\bar{v}$ corresponds to $\bar{\gamma}$. Through (11), the mean BEP expression is given by

$$
P_{\mathrm{b}}\left(\bar{v}, N_{\mathrm{p}} \varepsilon, M_{j}\right)=\left.P_{\mathrm{b}}(\bar{\gamma})\right|_{\bar{\gamma}=\frac{\bar{v}}{\frac{N_{\mathrm{p}}}{N_{\mathrm{tot}}}(\varepsilon-1)+1}}
$$

and the $j$-th SNR threshold $\left(M=M_{j}\right)$ is

$$
\bar{v}_{j}^{\star} \text { such that } P_{\mathrm{b}}\left(\bar{v}_{j}^{\star}, N_{\mathrm{p}} \varepsilon, M_{j}\right)=P_{\mathrm{b}}^{\star} \text {. }
$$

In the case of non-ideal channel estimation, the mean SNR is

$$
\breve{v} \triangleq \mathbb{E}\left\{|\widehat{h}|^{2}\right\} \frac{E}{N_{0}}=\bar{v}+\underbrace{\frac{1}{N_{\text {tot }}}+\frac{1-n_{\mathrm{p}}}{N_{\mathrm{p}} \varepsilon}}_{\Delta \bar{v}} .
$$

When ideal systems (ideal CSI without constraints) are considered, $\breve{v}$ corresponds to $\bar{\gamma}$.

For ideal CSI systems, the BEO is evaluated from (9) and (13), with $\chi=\bar{v}, \chi_{j}^{\star}=\bar{v}_{j}^{\star}$, and $F_{\chi}(\xi)=F_{\bar{v}}(\zeta)$, where $F_{\bar{v}}(\zeta)$ is the CDF of $\bar{v}$. For log-normal shadowing, $\bar{v}_{\mathrm{dB}}$ is Gaussian distributed with mean $\mu_{\mathrm{dB}}^{\prime}$ and variance $\sigma_{\mathrm{dB}}^{2}$, with CDF given by ${ }^{4}$

$$
F_{\bar{v}}(w)=Q\left(\frac{\mu_{\mathrm{dB}}^{\prime}-10 \log _{10}(w)}{\sigma_{\mathrm{dB}}}\right) .
$$

\footnotetext{
${ }^{4}$ When $\varepsilon=1, \mu_{\mathrm{dB}}^{\prime}=\mu_{\mathrm{dB}}$, while for $\varepsilon \neq 1$, the median SNR penalty becomes $\Delta \mu_{\mathrm{dB}} \triangleq \widehat{\mu}_{\mathrm{dB}, 0}^{\prime}-\mu_{\mathrm{dB}, 0}^{\prime}$.
} 
For non-ideal CSI systems, the BEO can be derived from (14) and (28) as

$$
\begin{aligned}
P_{\mathrm{o}} & =Q\left(a_{0}\right)+\sum_{j=1}^{J}\left[Q\left(a_{j}\right)-Q\left(b_{j}\right)\right], \\
\text { where } \quad a_{j} & =\frac{\mu_{\mathrm{dB}}^{\prime}-\bar{v}_{j, \mathrm{~dB}}^{\star}}{\sigma_{\mathrm{dB}}} \\
b_{j} & =\frac{\mu_{\mathrm{dB}}^{\prime}-10 \log _{10}\left(\bar{v}_{j}^{\star}-\frac{1}{N_{\mathrm{tot}}}-\frac{1-n_{\mathrm{p}}}{N_{\mathrm{p}} \varepsilon}\right)}{\sigma_{\mathrm{dB}}} .
\end{aligned}
$$

The ASE is evaluated from (18) and (19), with $\chi=\bar{v}, \chi_{j}^{\star}=$ $\bar{v}_{j}^{\star}$ and $F_{\chi}(\xi)=F_{\breve{v}}(x)$ is given by

$$
F_{\breve{v}}(x)=Q\left(\frac{\mu_{\mathrm{dB}}^{\prime}-10 \log _{10}\left(x-\frac{1}{N_{\mathrm{tot}}}-\frac{1-n_{\mathrm{p}}}{N_{\mathrm{p}} \varepsilon}\right)}{\sigma_{\mathrm{dB}}}\right)
$$

for $x \geq\left(1 / N_{\text {tot }}\right)+\left(1-n_{\mathrm{p}}\right) / N_{\mathrm{p}} \varepsilon$ and 0 otherwise.

\section{Numerical Results}

We now present analytical, mainly, and simulative results in terms of ASE and BEO for both FAM and SAM systems. The simulative results are intended to verify the analysis in block fading and compare SAM and FAM also in timevarying channels. We assume coherent detection of $M$-QAM with $M \in\left\{4,16,64\right.$, and 256\}, $N_{\mathrm{d}}$-branch MRD, and Gray code mapping. Composite Rayleigh fading and log-normal shadowing channels are considered with both ideal and nonideal channel estimation. ${ }^{5}$ For non-ideal channel estimation, the ASE is evaluated by (19), with a target BEP of $10^{-2}$ and a maximum BEO of $5 \%$ (typical values for uncoded systems). We denote by $K$ the ratio between the frame lengths with SAM and FAM (i.e., the ratio of the coherence time, for largeand small-scale fading), as

$$
K=\frac{N_{\text {tot }}^{\mathrm{SAM}}}{N_{\mathrm{tot}}^{\mathrm{FAM}}}
$$

We assume $N_{\text {tot }}^{\mathrm{FAM}}=100$ symbols and $K=1000$ [31][33] which provide a conservative comparison since for realistic shadowing and fading channels [32], [34]-[37], $K$ can be greater than 1000 and consequently the gain of SAM compared to FAM can be even higher than what shown in the results. We now provide an example. For time-varying channels, the coherence time of the small-scale fading is inversely proportional to the maximum Doppler frequency: at $900 \mathrm{MHz}$ the coherence time is about $72 \mathrm{~ms}$ and $4 \mathrm{~ms}$ for a mobile speed of $3 \mathrm{~km} / \mathrm{h}$ and $50 \mathrm{~km} / \mathrm{h}$, respectively. On the other hand, the coherence time of the shadowing is proportional to the coherence distance (e.g., $100-200 \mathrm{~m}$ in a suburban area and tens of meters in an urban area [38]). Assuming a coherence distance of $100 \mathrm{~m}$, this results in a coherence time of about $120 \times 10^{3} \mathrm{~ms}$ and $7.2 \times 10^{3} \mathrm{~ms}$ at $3 \mathrm{~km} / \mathrm{h}$ and $50 \mathrm{~km} / \mathrm{h}$, respectively. This would lead to $K=1600 \div 1800$. Assuming a symbol period of $66 \mu \mathrm{s}$ as for universal mobile telecommunications system (UMTS)[39], $N_{\text {tot }}^{\text {FAM }}=60 \div 1000$ symbols in a coherence time. ${ }^{6}$ In the following figures, we

\footnotetext{
${ }^{5}$ Without loss of generality, we assume $\sigma_{\mathrm{h}}^{2}=1 / 2$.

${ }^{6}$ For time-varying fading, the coherence time of the small-scale fading has been derived as $T_{\mathrm{c}}=9 /\left(16 \pi f_{\mathrm{D}}\right)$ [37], where $f_{\mathrm{D}}$ is the Doppler frequency.
}

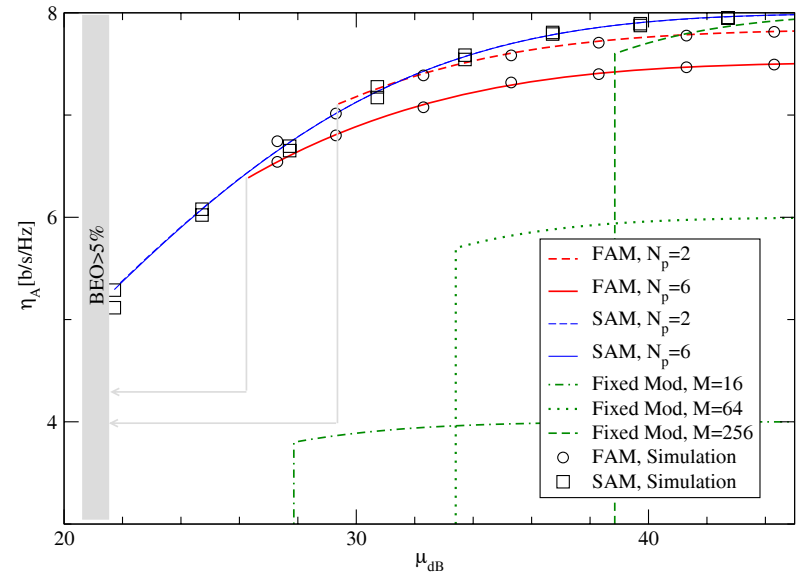

Fig. 2. ASE for SAM and FAM systems with non-ideal channel estimation: $M_{\max }=256$, dual-branch MRD, maximum BEO $5 \%, P_{\mathrm{b}}^{\star}=10^{-2}, \varepsilon=1$, $N_{\text {tot }}^{\mathrm{FAM}}=100, K=1000$, and $\sigma_{\mathrm{dB}}=8$. Comparison with fixed modulation systems $(M=16,64$ and 256$)$. Both analytical and simulative results are provided.

first illustrate a comparison between analytical and simulative results and we consider the block fading channels described in Section II.

In Fig. 2 the ASE of FAM, SAM, and fixed modulation systems is reported as a function of the mean SNR with $N_{\mathrm{d}}=2$ branches MRD, $M_{\max }=256, \varepsilon=1$ (i.e., $E_{\mathrm{p}}=E_{\mathrm{s}}$ ), and $\sigma_{\mathrm{dB}}=8 .^{7}$ For non-ideal channel estimation, SAM can outperform FAM, for both $N_{\mathrm{p}}=2$ and 6 , which confirm the importance of the analysis for design in practical systems. In particular it can be noticed that the lowest median SNR, for which the BEO requirement of $5 \%$ is satisfied, becomes advantageous for SAM as the channel estimation accuracy increases (i.e., $N_{\mathrm{p}}$ increases). Thus, SAM satisfies BEO requirements also in FAM-denied region (i.e., the $\mu_{\mathrm{dB}}$ region in which FAM systems experience a BEO greater than the target one). Then, by increasing $N_{\mathrm{p}}$ within the frame, the crossing point beyond which SAM outperforms FAM is for lower median SNR. Moreover, it should be noticed that both FAM and SAM provide a considerable gain in terms of ASE compared to the fixed modulation schemes. In this figure, both analytical and simulative results are provided, and their good match validates the analytical framework.

In Fig. 3, the comparison between FAM and SAM schemes is shown in terms of both ASE and BEO for ideal and nonideal channel estimation. The two systems with dual-branch MRD are compared for several maximum constellation values $\left(M_{\max }=4,16,64\right.$, and 256), pilot schemes $\left(N_{\mathrm{p}}=2\right.$, and 6 , and $\varepsilon=1$ ), and $\mu_{\mathrm{dB}}=20$, and 30 , with $\sigma_{\mathrm{dB}}=8$. For ideal channel estimation, by increasing the $M_{\max }$ value, the ASE increases while the BEO is constant to the value obtained for $M=4$; here, the FAM slightly outperforms SAM in terms of ASE. Conversely, when non-ideal channel estimation is considered, the BEO increases accordingly with the maximum constellation parameter due to the overestimation of the SNR in the feedback. In particular, the FAM systems have a non

\footnotetext{
${ }^{7}$ For fixed modulation systems, that does not require a feedback, ideal CSI is assumed.
} 


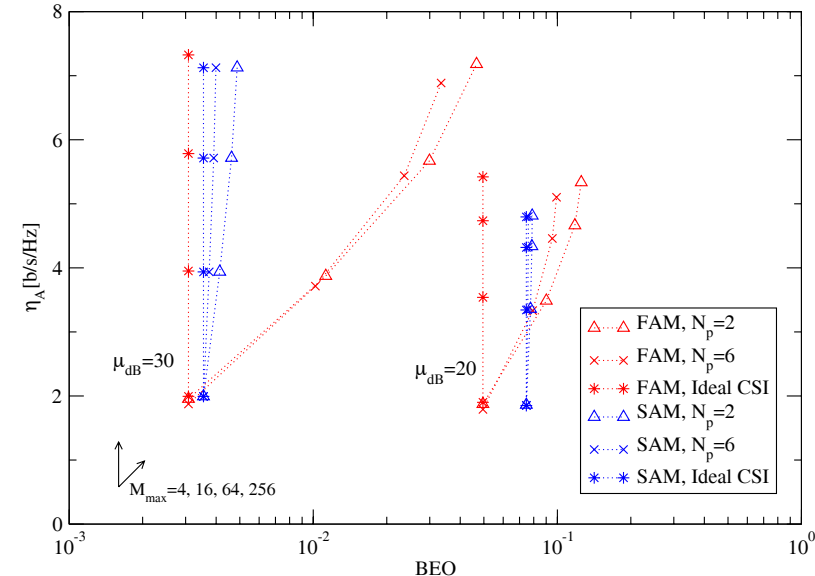

Fig. 3. ASE vs. BEO for FAM and SAM systems with $M_{\max }=$ $4,16,64$, and 256, dual-branch MRD, $\varepsilon=1, \sigma_{\mathrm{dB}}=8, \mu_{\mathrm{dB}}=20$, and $30, N_{\text {tot }}^{\text {FAM }}=100$, and $K=1000$. Results are evaluated for both ideal and non-ideal CSI (with $N_{\mathrm{p}}=1,2$, and 6).

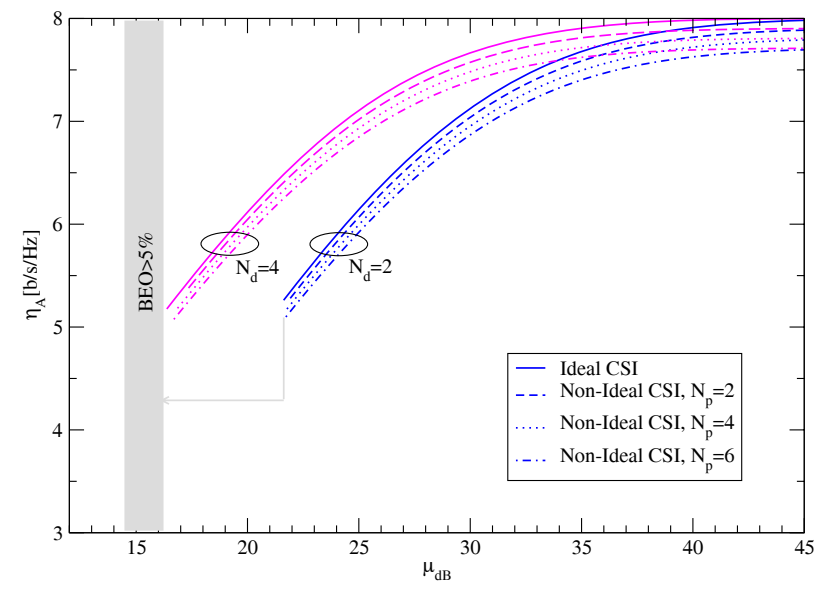

Fig. 4. ASE vs. $\mu_{\mathrm{dB}}$ for SAM systems with $\operatorname{MRD}\left(N_{\mathrm{d}}=2\right.$ and 4$), M_{\max }=$ 256 , maximum BEO $5 \%, P_{\mathrm{b}}^{\star}=10^{-2}, \varepsilon=1, \sigma_{\mathrm{dB}}=8$, and $N_{\mathrm{tot}}^{\mathrm{SAM}}=200$. Results are evaluated for both ideal and non-ideal channel estimation.

negligible increasing of the $\mathrm{BEO}$ value; for example, for $\mu_{\mathrm{dB}}=30 \mathrm{~dB}, M_{\max }=16$, and $N_{\mathrm{p}}=6$, a FAM system experiences a BEO of $10^{-2}$, despite the $3.5 \times 10^{-3}$ experienced by the SAM system. Also, for $\mu_{\mathrm{dB}}=30 \mathrm{~dB}$, the ASE of SAM is greater than that of FAM for both $N_{\mathrm{p}}=2$ and 6 .

The ASE as a function of $\mu_{\mathrm{dB}}$ is reported in Fig. 4 for SAM systems with MRD ( $N_{\mathrm{d}}=2$ and 4 branches $), M_{\max }=$ $256, \varepsilon=1, \sigma_{\mathrm{dB}}=8$, and several values of $N_{\mathrm{p}}$. Here, we consider $N_{\text {tot }}^{\mathrm{SAM}}=200$ symbols, which emphasizes more than in practice the effect of non-ideal CSI and pilot insertion on the system performance. The tradeoff between channel estimation quality and ASE can be observed in the figure; note that for the considered system, $N_{\mathrm{p}}=2$ provides a sufficient quality estimation, with ASE greater than that for $N_{\mathrm{p}}=4$ and 6 .

The effect of pilot energy (i.e., the effect of $\varepsilon$ ) is evaluated in Fig. 5. In this figure, the median SNR penalty $\Delta \mu_{\mathrm{dB}}$ as a function of $\varepsilon$ is reported for SAM systems with dualbranch MRD receivers, $M_{\max }=256, N_{\text {tot }}^{\mathrm{SAM}}=200$ symbols, target BEO of $5 \%$ and various $N_{\mathrm{p}}$ values. The numerical

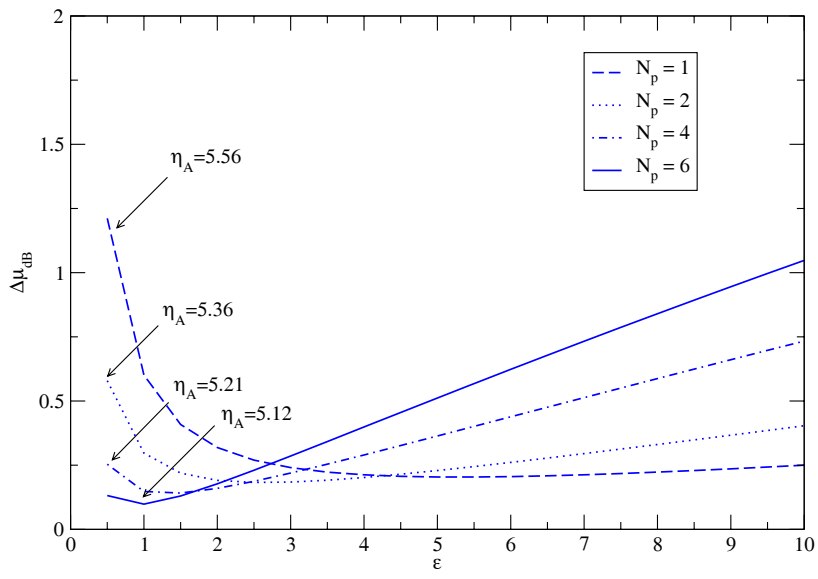

Fig. 5. $\Delta \mu_{\mathrm{dB}}$ vs. $\varepsilon$ for SAM systems with dual-branch MRD, $M_{\max }=256$, target BEO $5 \%, P_{\mathrm{b}}^{\star}=10^{-2}, \sigma_{\mathrm{dB}}=8, N_{\mathrm{p}}=1,2$, and 6 , and $N_{\text {tot }}^{\text {SAM }}=$ 200.

values reported represent the ASE evaluated for $\varepsilon=0.5$ (i.e., $E_{\mathrm{p}}=E_{\mathrm{s}} / 2$ ) and for those values of median SNR satisfying BEO requirements. Low median SNR penalty leads to performance close to that of ideal case. In particular, for each $N_{\mathrm{p}}$, an $\varepsilon$ value that minimizes the penalty can be obtained. By increasing $\varepsilon$ the channel estimation accuracy increases, while the symbol energy might decrease.

In Fig. 6 we provide the performance of SAM systems for various $N_{\text {tot }}$ values (i.e., several frame lengths). In particular, we compare the systems performance in terms of ASE (Fig. 6(a)) and in terms of BEO (Fig. 6(b)) as function of $n_{\mathrm{p}}$ for SAM systems with $M_{\max }=256$, four-branch MRD, $\varepsilon=1$, $\mu_{\mathrm{dB}}=20$ and 25 , and $\sigma_{\mathrm{dB}}=8$. Fig. $6\left(\right.$ a) shows the ASE vs. $n_{\mathrm{p}}$ when $N_{\text {tot }}$ is equal to $10^{2}, 10^{3}$, and $10^{4}$. For low $n_{\mathrm{p}}$ the system with the lowest number of pilot symbols (i.e., the system with $N_{\text {tot }}=10^{2}$ ) outperforms the others. From (7), it can be noticed that, the lower is $N_{\mathrm{p}}$, the higher is $\Delta \bar{\gamma}$. The overestimation of the mean SNR can achieve an ASE higher than the ideal systems, but it leads to an increasing of the BEO, as driven by (16). This drawback is depicted in Fig. 6(b), where the BEO as a function of $n_{\mathrm{p}}$ is provided. The greater the $N_{\mathrm{p}}$, the greater the $\Delta \bar{\gamma}$ and thus the greater is the BEO. For example, when $\mu_{\mathrm{dB}}=25$ and $n_{\mathrm{p}}=0.01$, the system with $N_{\text {tot }}=10^{2}$ (and thus $N_{\mathrm{p}}=1$ ) achieves a BEO of $2 \times 10^{-2}$ despite the $5 \times 10^{-3}$ and $3.5 \times 10^{-3}$ experienced by the systems with $N_{\text {tot }}=10^{3}$ and $10^{4}$, respectively.

A comparison between FAM and SAM performance in terms of the ASE penalty in (20) is provided in Fig. 7, for systems with dual-branch MRD, $M_{\max }=256$, target BEO $5 \%, P_{\mathrm{b}}^{\star}=10^{-2}, \sigma_{\mathrm{dB}}=8, N_{\mathrm{tot}}^{\mathrm{FAM}}=100$ and $K=1000$. Remember that the ASE penalty needs to be minimized and, in particular, $\Delta \eta$ approaches 1 for systems with $\eta_{\mathrm{A}}$ that tends to $\eta^{\text {(ideal) }}$. For both FAM and SAM, the penalty increases accordingly with the number of pilot symbols within each frame. Also, the penalty on the ASE for SAM is slightly lower than that of FAM.

The case of time-varying channel is considered in Fig. 8, where the ASE as a function of the median SNR is provided for FAM and SAM for dual-branch systems with non-ideal 


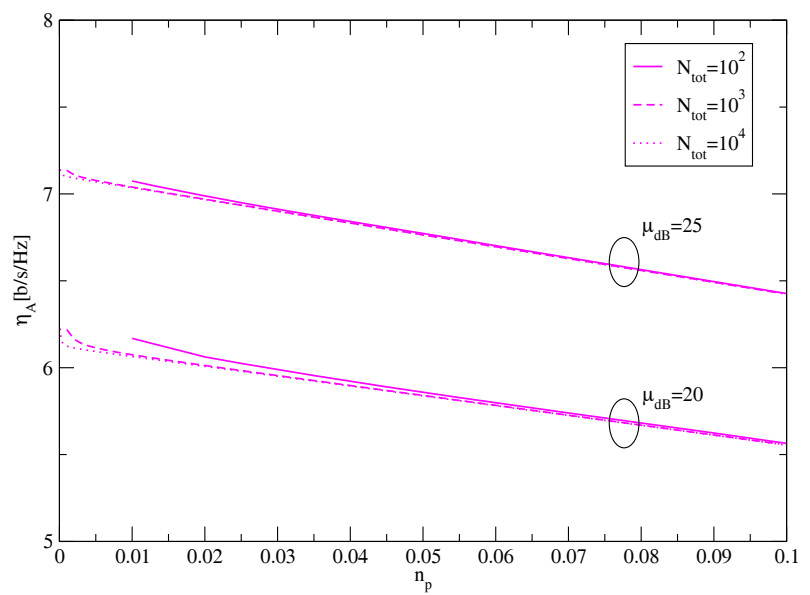

(a) ASE

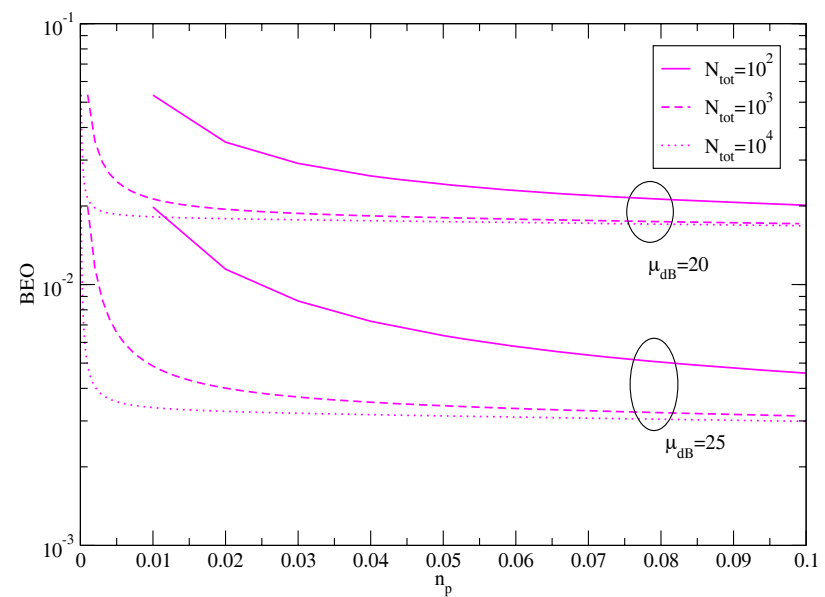

(b) BEO

Fig. 6. ASE and BEO vs. $n_{\mathrm{p}}$ for SAM systems with $M_{\max }=256$, four-branch MRD, $\varepsilon=1, \sigma_{\mathrm{dB}}=8, \mu_{\mathrm{dB}}=20$ and 25 , and $N_{\text {tot }}=10^{2}, 10^{3}$, and $10^{4}$.

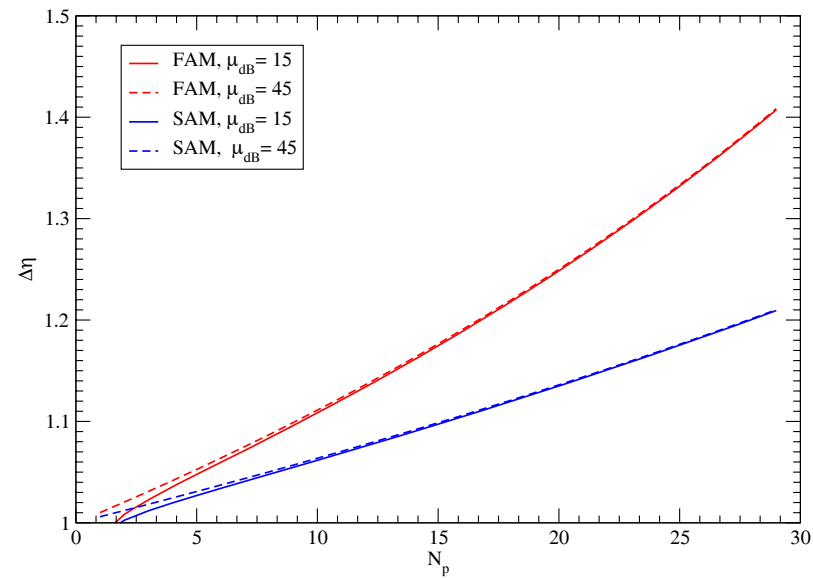

Fig. 7. $\Delta \eta$ vs. $N_{\mathrm{p}}$ for FAM and SAM systems with dual-branch MRD, $M_{\max }=256, P_{\mathrm{b}}^{\star}=10^{-2}, \sigma_{\mathrm{dB}}=8, N_{\mathrm{tot}}^{\mathrm{FAM}}=100, K=1000$, and non-ideal CSI.

CSI, $M_{\max }=256, \varepsilon=1, N_{\mathrm{p}}=6$, and $\sigma_{\mathrm{dB}}=8$. Timevarying channels are considered for several coherence times $(v=3,50$, and $110 \mathrm{~km} / \mathrm{h})$, and the simulative results are reported. As observed in the figure, also for time-varying fading channels, the SAM can outperform the FAM. Moreover, in the slow fading case, the performance degradation due to channel estimation errors is reduced, respect to fast fading channels. For example, at $50 \mathrm{~km} / \mathrm{h} \mathrm{SAM}$ achieves an ASE of $7.5[\mathrm{~b} / \mathrm{s} / \mathrm{Hz}]$ with $\mu_{\mathrm{dB}}=34$, despite the $\mu_{\mathrm{dB}}=38 \mathrm{~dB}$ required by the FAM. In addition to the ASE comparison, the SAM can outperform FAM in terms of BEO. The region of $\mu_{\mathrm{dB}}$ over which the SAM experiences an outage probability lower than or equal to the target BEO (in-service region) is wider than for FAM. This means that SAM systems are defined also in FAM-denied regions. Note also that, the higher is the velocity, the greater is the gap between the FAM and SAM in-service regions.

From the above results, the system designer can obtain the minimum value of the median SNR for specified target BEP,

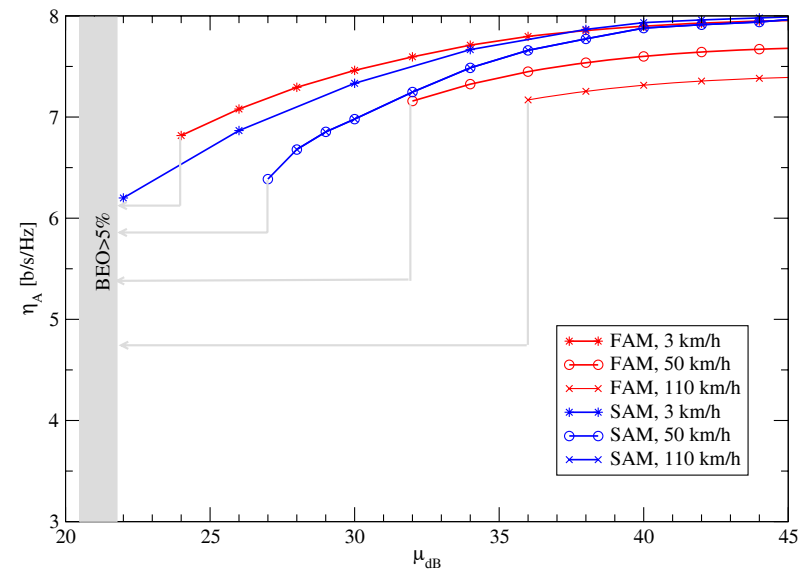

Fig. 8. ASE for SAM and FAM in systems with time-varying channel model with mobility of 3,50 , and $110 \mathrm{~km} / \mathrm{h}$ and with non-ideal channel estimation: $M_{\max }=256$, dual-branch MRD, maximum BEO $5 \%, P_{\mathrm{b}}^{\star}=10^{-2}, \varepsilon=1$, $K=1000, N_{\mathrm{p}}=6$ and $\sigma_{\mathrm{dB}}=8$. Simulative results are given.

BEO, and ASE in various channel conditions and diversity settings. Since the median SNR is tied to the propagation law and location of the user, one can design the wireless system (e.g., cell size, and power levels for cellular systems) that satisfies the requirements. Moreover, based on the imposed constraints, it is possible to understand the performance of both FAM and SAM in terms of ASE and BEO, and select the adaptive technique which is more suitable to fulfill the requirements.

\section{CONCLUSIONS}

We analyzed fast adaptive modulation (FAM) and slow adaptive modulation (SAM) systems with multichannel reception and non-ideal channel estimation under energy constraints. An appropriate figure of merit for the evaluation of the achieved SE (ASE) is defined. It takes into account the tradeoff between channel estimation and data reconstruction for a given total amount of energy per frame. The mathematical 
framework enables a system designer to evaluate the amount of energy and resources to be devoted to channel estimation for given target bit error probability and outage. Numerical results show that SAM systems, despite the lower feedback rate, can outperform the FAM systems and operate in FAM-denied regions. This gives a different prospective for the design of adaptive communication systems.

\section{ACKNOWLEDGMENT}

The authors wish to thank the editor and anonymous reviewers for helpful suggestions. The authors wish also to thank M. Z. Win, M. Chiani, O. Andrisano, and colleagues of NEWCom++ WPR.3 for helpful discussions.

\section{APPENDIX \\ Bit ERRor OUtAGE IN FAM SYSTEMS}

For FAM systems the $\Delta \chi=\Delta \gamma_{\mathrm{T}}$ is the RV resulting from the difference of two correlated chi-square distributed RVs

$$
\Delta \gamma_{\mathrm{T}}=\widehat{\gamma}_{\mathrm{T}}-\gamma_{\mathrm{T}}=\Delta h \frac{E_{\mathrm{s}}}{N_{0}}
$$

where

$$
\Delta h=\sum_{n=1}^{N_{\mathrm{d}}}\left(\left|\widehat{h}_{n}\right|^{2}-\left|h_{n}\right|^{2}\right) .
$$

and $\rho^{2}=\sigma_{\mathrm{h}}^{2} /\left(\sigma_{\mathrm{h}}^{2}+\sigma_{\mathrm{e}}^{2}\right)=\bar{\gamma} /\left(\bar{\gamma}+\left(N_{\mathrm{p}} \varepsilon\right)^{-1}\right)$ is the normalized correlation. As reported in [40], the PDF of $\Delta h$ can be expressed as (31) at the bottom of the page, where $\sigma_{1}^{2}=\sigma_{\mathrm{h}}^{2}$, $\sigma_{2}^{2}=\sigma_{\mathrm{h}}^{2}+\sigma_{\mathrm{e}}^{2}$, and

$$
\begin{aligned}
& \gamma^{-}=\frac{\left[\left(\sigma_{2}^{2}-\sigma_{1}^{2}+4 \sigma_{1}^{2} \sigma_{2}^{2}\left(1-\rho^{2}\right)\right)^{2}\right]^{1 / 2}}{\sigma_{1}^{2} \sigma_{2}^{2}\left(1-\rho^{2}\right)} \\
& \alpha^{ \pm}=\gamma^{-} \pm \frac{\sigma_{2}^{2}-\sigma_{1}^{2}}{\sigma_{1}^{2} \sigma_{2}^{2}\left(1-\rho^{2}\right)} .
\end{aligned}
$$

Thus, the PDF of $\Delta \gamma_{\mathrm{T}}$, conditioned to $\bar{\gamma}$ results

$$
f_{\Delta \gamma_{\mathrm{T}} \mid \bar{\gamma}}(\zeta)=\left.f_{\Delta h}(\xi)\right|_{\xi=\Delta \gamma_{\mathrm{T}} 2 \sigma_{\mathrm{h}}^{2} / \bar{\gamma}} \frac{2 \sigma_{\mathrm{h}}^{2}}{\bar{\gamma}} .
$$

The unconditioned PDF of the mean SNR can be written as

$$
f_{\Delta \gamma_{\mathrm{T}}}(\zeta)=\int_{-\infty}^{\infty} f_{\Delta \gamma_{\mathrm{T}} \mid \bar{\gamma}}(\zeta) f_{\bar{\gamma}}(\nu) d \nu
$$

Knowing the distribution of $\Delta \gamma_{\mathrm{T}}$, the unconditioned BEO for FAM systems can be evaluated.

\section{REFERENCES}

[1] J. K. Cavers, "Variable-rate transmission for Rayleigh fading channels," IEEE Trans. Commun., vol. COM-20, no. 1, pp. 15-22, Feb. 1972.

[2] W. T. Webb and R. Steele, "Variable rate QAM for mobile radio," IEEE Trans. Commun., vol. 43, no. 7, pp. 2223-2230, July 1995.

[3] A. J. Goldsmith and S.-G. Chua, "Variable-rate variable-power MQAM for fading channel," IEEE Trans. Commun., vol. 45, no. 10, pp. 12181230, Oct. 1997.

[4] S. T. Chung and A. J. Goldsmith, "Degree of freedom in adaptive modulation: a unified view," IEEE Trans. Commun., vol. 49, no. 9, pp. 1561-1571, Sep. 2001

[5] A. Conti, M. Z. Win, and M. Chiani, "Slow adaptive M-QAM with diversity in fast fading and shadowing," IEEE Trans. Commun., vol. 55, no. 5, pp. 895-905, May 2007.

[6] T. Keller and L. Hanzo, "Adaptive modulation techniques for duplex OFDM transmission," IEEE Trans. Veh. Technol., vol. 49, no. 5, pp. 1893-1906, Sep. 2000.

[7] A. Maaref and S. Aissa, "Adaptive modulation using orthogonal STBC in MIMO Nakagami fading channels," in Proc. IEEE Eighth International Symp. Spread Spectrum Techniques Appl., Sep. 2004, pp. 145-149.

[8] A. Conti, M. Z. Win, M. Chiani, and J. H. Winters, "Bit error outage for diversity reception in shadowing environment," IEEE Commun. Lett., vol. 7, no. 1, pp. 15-17, Jan. 2003.

[9] J. F. Paris, M. C. Aguayo-Torrse, and J. T. Entrambasaguas, "Impact of channel estimation error on adaptive modulation performance in flat fading," IEEE Trans. Commun., vol. 52, no. 5, pp. 716-720, May 2004.

[10] S. Ye, R. S. Blum, and J. L. J. Cimini, "Adaptive OFDM systems with imperfect channel state information," IEEE Trans. Wireless Commun., vol. 5, no. 11, pp. 3255-3265, Nov. 2006.

[11] S. Zhou and G. B. Giannakis, "How accurate channel prediction needs to be for transmit-beamforming with adaptive modulation over Rayleigh MIMO channels?" IEEE Trans. Wireless Commun., vol. 3, no. 4, pp. 1285-1294, July 2004

[12] J. Cavers, "An analysis of pilot symbol assisted modulation for Rayleigh fading channels," IEEE Trans. Veh. Technol., vol. 40, no. 4, pp. 686-693, Nov. 1991.

[13] W. M. Gifford, M. Z. Win, and M. Chiani, "Diversity with practical channel estimation," IEEE Trans. Wireless Commun., vol. 4, no. 4, pp. 1935-1947, July 2005.

[14] Y. Chen and N. C. Beaulieu, "Optimum pilot symbol assisted modulation," IEEE Trans. Commun., vol. 55, no. 8, pp. 1536-1546, Aug. 2007.

[15] A. Maaref and S. Aissa, "Optimized rate-adaptive PSAM for MIMO MRC systems with transmit and receive CSI imperfections," IEEE Trans. Commun., vol. 57, no. 3, pp. 821 -830, Mar. 2009.

[16] M. Chiani, A. Conti, and C. Fontana, "Improved performance in TDCDMA mobile radio system by optimizing energy partition in channel estimation," IEEE Trans. Commun., vol. 51, no. 3, pp. 352-355, Mar. 2003.

[17] R. McEliece and W. Stark, "Channels with block interference," IEEE Trans. Inf. Theory, vol. 30, no. 1, pp. 44-53, Jan. 1984.

[18] M. Chiani, "Error probability for block codes over channels with block interference," IEEE Trans. Inf. Theory, vol. 44, no. 7, pp. 2998-3008, Nov. 1998.

[19] M. Chiani, A. Conti, and O. Andrisano, "Outage evaluation for slow frequency-hopping mobile radio systems," IEEE Trans. Commun., vol. 47, no. 12, pp. 1865-1874, Dec. 1999.

[20] C. Xiao, Y. R. Zheng, and N. C. Beaulieu, "Second-order statistical properties of the WSS Jakes' fading channel simulator," IEEE Trans. Commun., vol. 50, no. 6, pp. 888-891, June 2002.

[21] Y. R. Zheng and C. Xiao, "Simulation models with correct statistical properties for Rayleigh fading channels," IEEE Trans. Commun., vol. 51, no. 6, pp. 920-928, June 2003.

[22] A. Conti, M. Z. Win, and M. Chiani, "On the inverse symbol-error probability for diversity reception," IEEE Trans. Commun., vol. 51, no. 5, pp. 753-756, May 2003.

[23] P. Mary, M. Dohler, J. M. Gorce, G. Villemaud, and M. Arndt, "BPSK bit error outage over Nakagami-m fading channels in lognormal

$$
f_{\Delta h}(\xi)= \begin{cases}\frac{|\xi|^{N_{\mathrm{d}}-1}}{\left(N_{\mathrm{d}}-1\right) !\left[2 \sigma_{1}^{2} \sigma_{2}^{2}\left(1-\rho^{2}\right) \gamma^{-}\right]_{\mathrm{d}}^{N}} \exp \left[\frac{1}{4} \alpha^{-} \xi\right] \sum_{i=0}^{N_{\mathrm{d}}-1} \frac{(m+i-1) !}{i !(m-i-1) !}\left(\frac{2}{\gamma^{-}|\xi|}\right)^{i} & \xi<0 \\ \frac{|\xi|^{N_{\mathrm{d}}-1}}{\left(N_{\mathrm{d}}-1\right) !\left[2 \sigma_{1}^{2} \sigma_{2}^{2}\left(1-\rho^{2}\right) \gamma^{-}\right]_{\mathrm{d}}^{N}} \exp \left[-\frac{1}{4} \alpha^{+} \xi\right] \sum_{i=0}^{N_{\mathrm{d}}-1} \frac{(m+i-1) !}{i !(m-i-1) !}\left(\frac{2}{\gamma^{-}|\xi|}\right)^{i} & \xi \geq 0\end{cases}
$$


shadowing environments," IEEE Commun. Lett., vol. 11, no. 7, pp. 565567, July 2007.

[24] A. Conti, W. M. Gifford, M. Z. Win, and M. Chiani, "Optimized simple bounds for diversity systems," IEEE Trans. Commun., vol. 57, no. 9, pp. 2674-2685, Sep. 2009.

[25] K. Cho and D. Yoon, "On the general BER expression of one- and twodimensional amplitude modulations," IEEE Trans. Commun., vol. 50, no. 7, pp. 1074-1080, July 2002.

[26] A. Conti, M. Z. Win, and M. Chiani, "Invertible bounds for $M$-QAM in fading channels," IEEE Trans. Wireless Commun., vol. 4, no. 5, pp. 1994-2000, Sep. 2005.

[27] M. Z. Win and J. H. Winters, "Analysis of hybrid selection/maximalratio combining in Rayleigh fading," IEEE Trans. Commun., vol. 47, no. 12, pp. 1773-1776, Dec. 1999.

[28] _ - "Virtual branch analysis of symbol error probability for hybrid selection/maximal-ratio combining in Rayleigh fading," IEEE Trans. Commun., vol. 49, no. 11, pp. 1926-1934, Nov. 2001.

[29] M. Z. Win, N. C. Beaulieu, L. A. Shepp, J. B. F. Logan, and J. H. Winters, "On the SNR penalty of MPSK with hybrid selection/maximal ratio combining over i.i.d. Rayleigh fading channels," IEEE Trans. Commun., vol. 51, no. 6, pp. 1012-1023, June 2003.

[30] W. M. Gifford, M. Z. Win, and M. Chiani, "Antenna subset diversity with non-ideal channel estimation," IEEE Trans. Wireless Commun., vol. 7, no. 5, pp. 1527-1539, May 2008.

[31] V. Erceg, L. J. Greenstein, S. Y. Tjandra, S. R. Parkoff, A. Gupta, B. Kulic, A. A. Julius, and R. Bianchi, "An empirically based path loss model for wireless channels in suburban environments," IEEE $J$. Sel. Areas Commun., vol. 17, no. 7, pp. 1205-1211, July 1999.

[32] W. C. Jakes, Microwave Mobile Communications. IEEE Press, 1995.

[33] W. L. Li, Y. J. Zhang, A. M.-C. So, and M. Z. Win, "Slow adaptive OFDMA through chance constrained programming," IEEE Trans. Signal Process., vol. 58, pp. 3858-3869, July 2010.

[34] M. Gudmundson, "Correlation model for shadow fading in mobile radio systems," Electron. Lett., vol. 27, no. 23, pp. 2145-2146, Nov. 1991.

[35] J. Fuhl, A. F. Molisch, and E. Bonek, "Unified channel model for mobile radio systems with smart antennas," IEE Proc. Radar, Sonar Navigation, vol. 145, no. 1, pp. 32-41, Feb. 1998.

[36] A. J. Goldsmith, L. J. Greenstein, and G. J. Foschini, "Error statistics of real-time power measurements in cellular channels with multipath and shadowing," IEEE Trans. Veh. Technol., vol. 43, no. 3, pp. 439-446, Aug. 1994.

[37] G. L. Stuber, Principles of Mobile Communication, 2nd edition. Kluwer Academic Publishers, 2001

[38] A. Duel-Hallen, S. Hu, and H. Hallen, "Long-range prediction of fading signals," IEEE Signal Process. Mag., vol. 17, no. 3, pp. 62-75, May 2000.

[39] Universal Mobile Telecommunications System (UMTS) standard, 3GPP TS 25.211; Physical channels and mapping of transport channels onto physical channels (FDD), version 8.5.0 Release 8, Sep. 2009.

[40] M. K. Simon, Probability Distributions Involving Gaussian Random Variables: A Handbook for Engineers and Scientists, 2nd edition. Springer, 2006.

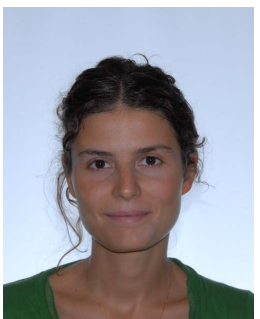

Laura Toni (S'06-M'09) received M.S. degree (with honors) in electrical engineering and the Ph.D. degree in electronics, computer science and telecommunications engineering from the University of Bologna, Italy, in 2005 and 2009, respectively..

In 2005, she joined the Department of Electronics, Informatics and Systems at the University of Bologna, to develop her research activity in the area of wireless communications. During 2007, she had been a visiting scholar at the University of California at San Diego, CA, working on video processing over wireless systems. Since 2009, she has been a frequent visitor to the UCSD, working on joint source and channel coding on wireless communication systems.

In June 2009, she joined the TeleRobotics and Applications (TERA) department at the Italian Institute of Technology (IIT), Genova, Italy. Her research interests are in the areas of image and video processing, wireless communications, and underwater communications.

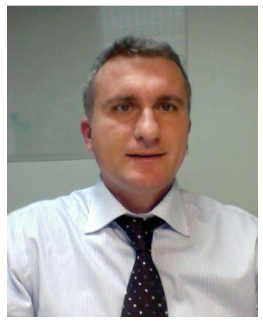

Andrea Conti (S'99-M'01-SM'11) received the Laurea degree (summa cum laude) in telecommunications engineering and the Ph.D. degree in electronic engineering and computer science from the University of Bologna, Italy, in 1997 and 2001, respectively.

In 2005, he joined the University of Ferrara, Italy, where he is an Assistant Professor. He was Researcher with Consorzio Nazionale Interuniversitario per le Telecomunicazioni (CNIT) from 1999 to 2002 and with Istituto di Elettronica e di Ingegneria dell'Informazione e delle Telecomunicazioni, Consiglio Nazionale delle Ricerche (IEIIT/CNR) from 2002 to 2005 at the Research Unit of Bologna. In Summer 2001, he was with the Wireless Systems Research Department at AT\&T Research Laboratories. Since 2003, he has been a frequent visitor to the Wireless Communication and Network Sciences Laboratory at the Massachusetts Institute of Technology (MIT), where he presently holds the Research Affiliate appointment. He is a coauthor of Wireless Sensor and Actuator Networks: Enabling Technologies, Information Processing and Protocol Design (Elsevier, 2008). His research interests involve theory and experimentation of wireless systems and networks including network localization, adaptive diversity communications, cooperative relaying techniques, interference management, and sensor networks.

Dr. Conti is currently an Editor for the IEEE COMMUNICATIONS LETTERS. He served as an Editor for the IEEE TRANSACTIONS ON WIRELESS COMMUNICATIONS from 2003 to 2009 and Guest-Editor for the EURASIP Journal on Advances in Signal Processing (Special Issue on Wireless Cooperative Networks) in 2008. He is the Technical Program Chair for the IEEE International Conference on Ultra-Wideband 2011; Technical Program Co-Chair for the IEEE Vehicular Technology Conference 2011-Spring and the Wireless Communications Symposium of IEEE Global Communications Conference 2010; and Technical Program Vice-Chair for the IEEE Wireless Communications and Networking Conference 2009. He served as reviewer for IEEE and IET journals and as Technical Program Committee Member for numerous IEEE conferences. He is elected Vice-Chair and Secretary of the IEEE Communications Society's Radio Communications Technical Committee for the terms 2011-2012 and 2009-2010, respectively. 